\title{
Chemical constituents of the genus Trichosanthes (Cucurbitaceae) and their biological activities: A review
}

\author{
Wachirachai Pabuprapap, Apichart Suksamrarn* \\ Department of Chemistry and Center of Excellence for Innovation in Chemistry, Faculty of Science, \\ Ramkhamhaeng University, Bangkok 10240 Thailand \\ *Corresponding author, e-mail: asuksamrarn@yahoo.com,s_apichart@ru.ac.th
}

Received 11 May 2021

Accepted 31 May 2021

\begin{abstract}
Trichosanthes is one of the largest genera in the Cucurbitaceae family. It is constantly used in traditional medications to cure diverse human diseases and is also utilized as ingredients in some food recipes. It is enriched with a diversity of phytochemicals and a wide range of biological activities. The major chemical constituents in this plant genus are steroids, triterpenoids and flavonoids. This review covers the different types of chemical constituents and their biological activities from the Trichosanthes plants.
\end{abstract}

KEYWORDS: Trichosanthes, Cucurbitaceae, phytochemistry, chemical constituent, biological activity

\section{INTRODUCTION}

Natural products have long been and will continue to be extremely important as the most promising source of biologically active compounds for the treatment of human and animal illness and disorder. They broadly present in natural sources, including microorganisms, marines, animals and especially plants [1]. Recent investigations in phytochemistry and biological activities have resulted in the isolation and biological activity assessments of various new bioactive compounds from different plant genus. Also, plant-derived natural products have played a critical role in drug discovery by enormous scaffold variety and structural complexity, which can produce significant quantities of bioactive compounds, including alkaloids, anthraquinones, flavonoids, polyketides and terpenoids, which were reported to possess promising pharmacological activities [2].

The Cucurbitaceae family, called cucurbits or the gourd family, is the family of plants consisting of about 125 genera and 1000 species widely distributed throughout the tropics and temperate areas around the world [3]. This plant family is dioecious or rarely monoecious, annual or perennial, herbaceous with climbing or trailing stems bearing tendrils and often arising from woody rootstock [4]. Some of the important genera belonging to the family are Trichosanthes, Luffa, Lagenaria, Benincasa, Momordica, Cucurbita, Cucumis and Citrullus [5].
Cucurbitaceae plants are widely used in traditional medicines for a variety of ailments, especially in the ayurvedic and Chinese medicines, including treatments against gonorrhoea, ulcers, respiratory diseases, jaundice, syphilis, scabies, constipation, worms, piles, leprosy, skin infections, haemoptysis, diabetes, night-blindness, obesity, kidney and liver diseases [6-8]. In modern medicine, the Cucurbitaceae comprises plants of great interest, with a wide range of biological activities including antidiabetic, anti-tumoral, anti-parasitic, anti-bacterial, anti-inflammatory and cytotoxic activities [9].

The genus Trichosanthes belongs to the Cucurbitaceae family, the largest genus of approximately 100 species worldwide, widely distributed in Southern and Eastern Asia, Australia and Islands of the western Pacific [10]. At least 25 species in this genus have been found distributed throughout Thailand [11]. Some of them are grown commercially for their fleshy fruits used as vegetables, most popular in South and Southeast Asia. In addition, the plants of this genus are commonly used in Asian folk medicine to treat a wide range of biological activities including anti-inflammatory, anti-diabetic, anti-ulcer and cardioprotective activities [12]. This review focuses on chemical constituents and biological properties of Trichosanthes species and their prospects for improved usage in medicinal applications. The chemical constituents and biological activities of the selected 10 species from the genus Trichosanthes are summarized in Fig. 1. 


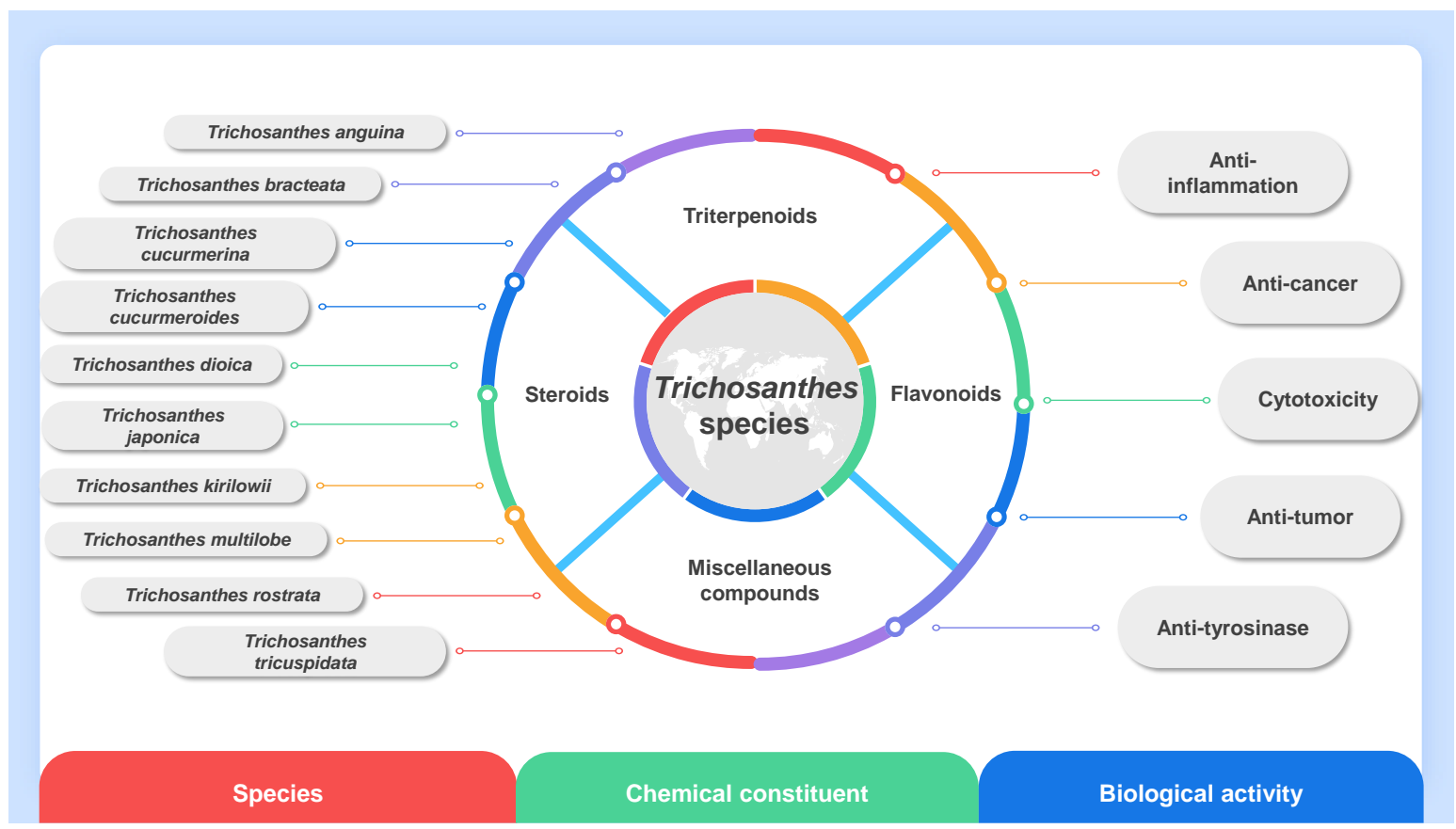

Fig. 1 Summary of the selected species from the genus Trichosanthes, their chemical constituents and biological activities.

\section{CHEMICAL CONSTITUENTS AND BIOLOGICAL ACTIVITIES}

A total of 103 compounds including 15 steroids (compounds 1-15), 55 triterpenoids classified into 30 cucurbitacin triterpenoids (compounds 16-45), 4 cycloartane triterpenoids (compounds 46-49) and 21 pentacyclic triterpenoids (compounds 50-70), 9 flavonoids (compounds 71-79), and 24 other compounds (compounds 80-103) were identified from Trichosanthes plants. Most of them have been studied for a variety of biological activities. The chemical structures of the isolated compounds from Trichosanthes species are shown in Figs. 2-5. The list of compound names and their biological activities as well as their structure classifications are presented in Table 1. Some selected biological activities are highlighted in a separate topic.

\section{Steroids and triterpenoids}

Fifteen steroids (1-15) were isolated from T. cucumerina, T. cucumeroides, T. japonica, T. kirilowii and T. tricuspidata (Fig. 2) [13-21]. Among several steroids, compound $\mathbf{2}$ markedly showed inhibitory effects on TPA-induced inflammation in mice. The 50\% inhibitory dose of 2 for $12-O$ tetradecanoylphorbol-13-acetate (TPA)-induced inflammation was $0.7 \mathrm{mg} /$ ear, while the inhibitory effect of compound $\mathbf{1}$ was weaker than that of 2 [13].

Thirty cucurbitacin triterpenoids (16-45) were isolated from T. cucumerina, T. kirilowii and T. tricuspidata (Fig. 3) and have been reported for different biological activities [18,22-29]. A number of publications revealed that cucurbitacins are the constituents of Trichosanthes species. Cucurbitacins which have the structurally diverse steroidal triterpenoid skeleton found in the plant members of Cucurbitaceae and several plant families possessed extensive pharmacological potential against inflammation, cancer, atherosclerosis and diabetes. However, cucurbitacins are not usually utilized as medicinal agents because of their toxicity. Compound $\mathbf{3 1}$ has been reported to display significant cytotoxicity against KB cell line [28]. The chemical modification of various functional groups of cucurbitacins to reduce toxic effects may provide important lead compounds for future research.

Four cycloartane triterpenoids (46-49) have been isolated from T. kirilowii [30] and T. tricuspidata [31], whereas twenty-one pentacyclic triterpenoids (50-70) have been isolated from T. cucumerina, T. cucumeroides, T. dioica, T. kirilowii and T. truncata (Fig. 3) [19, 20, 32-40]. Some of these compounds were evaluated for their biological activities. For example, a marked inhibitory activity 
Table 1 Chemical constituents of Trichosanthes plants and their biological activities.

\begin{tabular}{|c|c|c|c|c|}
\hline Compound & Part of plant & Source & Biological activity & Ref. \\
\hline \multicolumn{5}{|l|}{ Steroids } \\
\hline $10 \alpha$-Cucurbitadienol (1) & Seeds & T. kirilowii & Anti-inflammation & [13] \\
\hline 7-Oxo-10 $\alpha$-cucurbitadienol (2) & Seeds & T. kirilowii & Anti-inflammation & [13] \\
\hline$\beta$-Sitosterol (3) & Leaves & T. cucumerina & - & [14] \\
\hline$\beta$-Sitosterol-D-glucoside (4) & Leaves & T. cucumerina & - & [14] \\
\hline \multirow[t]{5}{*}{$\alpha$-Spinasterol (5) } & Fruits & $\begin{array}{l}\text { T. cucumeroides } \\
\text { T. japonica }\end{array}$ & - & [15] \\
\hline & Roots & T. japonica & - & [16] \\
\hline & Roots & $\begin{array}{l}\text { T. japonica } \\
\text { T. kirilowii } \\
\text { T. cucumeroides }\end{array}$ & - & [17] \\
\hline & Unripe fruits & T. cucumerina & _- & [18] \\
\hline & Roots & T. cucumerina & - & [19] \\
\hline$\alpha$-Spinasterol acetate (6) & Seeds & T. japonica & - & [17] \\
\hline$\alpha$-Spinasterol-3-O- $\beta$-D-glucopyranoside (7) & Seeds & T. japonica & - & [17] \\
\hline \multirow[t]{4}{*}{ Stigmast-7-en-3 $\beta$-ol $(8)^{\mathrm{a}}$} & Roots & T. japonica & - & {$[16]$} \\
\hline & Seeds & T. japonica & - & [17] \\
\hline & Fruits & $\begin{array}{l}\text { T. japonica } \\
\text { T. cucumeroides }\end{array}$ & - & [20] \\
\hline & Roots & $\begin{array}{l}\text { T. japonica } \\
\text { T. kirilowii } \\
\text { T. cucumeroides }\end{array}$ & - & [17] \\
\hline \multirow[t]{3}{*}{$24 \alpha$-Ethyl-5 $\alpha$-cholesta-7,22-dien-3 $\beta$-ol (9) } & Fruits & $\begin{array}{l}\text { T. japonica } \\
\text { T. cucumeroides }\end{array}$ & - & [15] \\
\hline & Roots & T. japonica & - & [16] \\
\hline & Seeds & T. japonica & - & [17] \\
\hline \multirow{3}{*}{$24 \alpha$-Ethyl-5 $\alpha$-cholesta-7-en-3 $\beta$-ol (10) } & Fruits & T. cucumeroides & - & [15] \\
\hline & Roots & T. japonica & - & [16] \\
\hline & Seeds & T. japonica & - & [17] \\
\hline $24 \beta$-Ethyl-5 $\alpha$-cholesta-7,22,25-trien-3 $\beta$-ol (11) & Unripe fruits & T. cucumerina & - & [18] \\
\hline $\begin{array}{l}\text { 3-O- } \beta \text {-Glucopyranosyl- } 24 \xi \text {-ethyl- } 5 \alpha \text {-cholesta-7,22-dien- } \\
3 \beta \text {-ol (12) }\end{array}$ & Unripe fruits & T. cucumerina & - & [18] \\
\hline $24 \xi$-Ethyl- $5 \alpha$-cholesta-7-en-3 $\beta$-ol (13) & Unripe fruits & T. cucumerina & - & [18] \\
\hline Trichosanhemiketal A (14) & Roots & T. kirilowii & - & [21] \\
\hline Trichosanhemiketal B (15) & Roots & T. kirilowii & - & [21] \\
\hline \multicolumn{5}{|l|}{ Triterpenoids } \\
\hline \multirow[t]{4}{*}{ Cucurbitacin B (16) } & Fruit juice & T. cucumerina & $\begin{array}{l}\text { Cytotoxicity against } \\
\text { HeLa cell line }\end{array}$ & [22] \\
\hline & Unripe fruits & T. cucumerina & - & [18] \\
\hline & Roots & T. kirilowii & Anti-tumor & [23] \\
\hline & Roots & T. kirilowii & Anti-inflammation & [24] \\
\hline \multirow[t]{3}{*}{ Cucurbitacin D (17) } & Roots & T. kirilowii & Anti-tumor & [23] \\
\hline & Roots & T. kirilowii & Anti-inflammation & [24] \\
\hline & Roots & T. kirilowii & Anti-tyrosinase & [21] \\
\hline 2-O-ß-D-Glucopyranosyl-cucurbitacin D (18) & Roots & T. kirilowii & Anti-inflammation & [24] \\
\hline 2-O- $\beta$-D-Glucopyranosyl-cucurbitacin B (19) & Roots & T. kirilowii & $\begin{array}{l}\text { Cytotoxicity against } \\
\text { A-549, HT-29, OVCAR } \\
\text { and MCF-7 cell lines }\end{array}$ & [25] \\
\hline 2-epi-O-ß-D-Glucopyranosyl-cucurbitacin B (20) & Fruits & T. kirilowii & - & [26] \\
\hline \multirow[t]{2}{*}{ Isocucurbitacin D (21) } & Roots & T. kirilowii & Anti-tumor & [23] \\
\hline & Roots & T. kirilowii & Anti-inflammation & [24] \\
\hline Isocucurbitacin B (22) & Roots & T. kirilowii & Anti-tumor & [23] \\
\hline Dihydrocucurbitacin B (23) & Fruit juice & T. cucumerina & - & [27] \\
\hline 2-O- $\beta$-D-Glucopyranosyl-23,24-dihydrocucurbitacin B (24) & Unripe fruit & T. cucumerina & - & [18] \\
\hline 23,24-Dihydrocucurbitacin D (25) & Unripe fruit & T. cucumerina & - & [18] \\
\hline Cucurbitacin E (26) & Roots & T. kirilowii & Anti-inflammation & [24] \\
\hline \multirow[t]{2}{*}{ Cucurbitacin J 2-O- $\beta$-glucopyranoside (27) } & Fruit pericarps & T. tricuspidata & $\begin{array}{l}\text { Cytotoxicity against } \\
\mathrm{KB} \text { cell line }\end{array}$ & [28] \\
\hline & Fruits & T. tricuspidata & - & [29] \\
\hline Cucurbitacin K 2-O- $\beta$-glucopyranoside (28) & Fruits & T. tricuspidata & - & [29] \\
\hline Bryoamaride (29) & Fruits & T. tricuspidata & - & [29] \\
\hline 25-O-Acetyl-bryoamaride (30) & Fruits & T. tricuspidata & - & [29] \\
\hline Tricuspidatin (31) & Fruit pericarps & T. tricuspidata & $\begin{array}{l}\text { Cytotoxicity against } \\
\text { KB cell line }\end{array}$ & [28] \\
\hline Khekadaengoside A (32) & Fruits & T. tricuspidata & - & [29] \\
\hline Khekadaengoside B (33) & Fruits & T. tricuspidata & - & [29] \\
\hline Khekadaengoside C (34) & Fruits & T. tricuspidata & - & [29] \\
\hline Khekadaengoside D (35) & Fruits & T. tricuspidata & - & [29] \\
\hline Khekadaengoside E (36) & Fruits & T. tricuspidata & - & [29] \\
\hline Khekadaengoside F (37) & Fruits & T. tricuspidata & - & [29] \\
\hline Khekadaengoside G (38) & Fruits & T. tricuspidata & - & [29] \\
\hline Khekadaengoside H (39) & Fruits & T. tricuspidata & - & [29] \\
\hline
\end{tabular}

a This compound should be the same as "22-dihydro- $\alpha$-spinasterol" isolated from T. truncata [40]. However, no detailed information was available from [40]. 
Table 1 (Continued.)

\begin{tabular}{|c|c|c|c|c|}
\hline Compound & Part of plant & Source & Biological activity & Ref. \\
\hline Khekadaengoside I (40) & Fruits & T. tricuspidata & - & [29] \\
\hline Khekadaengoside J (41) & Fruits & T. tricuspidata & - & [29] \\
\hline Khekadaengoside K (42) & Fruits & T. tricuspidata & - & {$[29]$} \\
\hline Khekadaengoside L (43) & Fruits & T. tricuspidata & - & {$[29]$} \\
\hline Khekadaengoside M (44) & Fruits & T. tricuspidata & - & {$[29]$} \\
\hline Khekadaengoside N (45) & Fruits & T. tricuspidata & - & {$[29]$} \\
\hline Isocyclokirilodiol (46) & Seeds & T. kirilowii & - & {$[30]$} \\
\hline Cyclotricuspidoside A (47) & Leaves and stems & T. tricuspidata & - & {$[31]$} \\
\hline Cyclotricuspidoside B (48) & Leaves and stems & T. tricuspidata & - & {$[31]$} \\
\hline Cyclotricuspidoside C (49) & Leaves and stems & T. tricuspidata & - & {$[31]$} \\
\hline Bryonolol (50) & Seeds & T. kirilowii & - & {$[32]$} \\
\hline Bryonolol diacetate (51) & Seeds & T. kirilowii & Anti-inflammation & {$[32]$} \\
\hline 7-Oxoisomultiflorenol (52) & Seeds & T. kirilowii & Anti-inflammation & {$[32]$} \\
\hline 3-epi-Bryonolol (53) & Seeds & T. kirilowii & Anti-inflammation & {$[32]$} \\
\hline 3-epi-Bryonolol diacetate (54) & Seeds & T. kirilowii & Anti-inflammation & {$[32]$} \\
\hline \multirow[t]{2}{*}{ 7-Oxodihydrokarounidiol (55) } & Seeds & T. kirilowii & - & {$[33,34]$} \\
\hline & Seeds & T. cucumeroides & - & {$[35]$} \\
\hline 7-Oxodihydrokarounitriol (56) & Seeds & T. cucumeroides & - & {$[35]$} \\
\hline 7,11-Dioxodihydroxykarounidiol (57) & Seeds & T. cucumeroides & - & {$[35]$} \\
\hline Karounitriol (58) & Seeds & T. kirilowii & - & {$[34]$} \\
\hline $3 \beta$-Hydroxy-olean-13(18)-ene-28-oic acid (59) & Roots & T. cucumerina & - & {$[19]$} \\
\hline 3-Oxo-olean-13(18)-ene-30-oic acid (60) & Roots & T. cucumerina & $\begin{array}{l}\text { Cytotoxicity against } \\
\text { leukemia cell line }\end{array}$ & [19] \\
\hline 7-Oxo-8 $\beta$-D:C-friedo-olean-9(11)-ene-3 $\alpha, 29$-diol (61) & Seeds & T. kirilowii & Anti-inflammation & [32] \\
\hline 7-Oxo-8 $\beta$-D:C-friedo-olean-9(11)-ene-3 $\alpha, 29$-diol diacetate (62) & Seeds & T. kirilowii & Anti-inflammation & {$[32]$} \\
\hline 7-Oxodihydroxykarounidiol-3-O-benzoate (63) & Seeds & $\begin{array}{l}\text { T. cucumeroides } \\
\text { T. dioica }\end{array}$ & - & {$[36]$} \\
\hline \multirow[t]{2}{*}{ Karounidiol (64) } & Seeds & T. kirilowii & - & {$[34,37]$} \\
\hline & Seeds & T. kirilowii & $\begin{array}{l}\text { Anti-tumor } \\
\text { Cytotoxicity against } \\
\text { cancer cell line }\end{array}$ & {$[38]$} \\
\hline Karounidiol 3-O-benzoate (65) & Seeds & T. kirilowii & - & [37] \\
\hline \multirow[t]{2}{*}{ 3-Epikarounidiol (66) } & Seeds & T. kirilowii & - & {$[32]$} \\
\hline & Seeds & T. kirilowii & $\begin{array}{l}\text { Anti-inflammation } \\
\text { Anti-tumor }\end{array}$ & {$[38]$} \\
\hline 3-Epikarounidiol diacetate (67) & Seeds & T. kirilowii & Anti-inflammation & {$[32]$} \\
\hline Isokarounidiol (68) & Seeds & T. kirilowii & - & {$[34,39]$} \\
\hline \multirow[t]{3}{*}{ 5-Dehydrokarounidiol (69) } & Seeds & T. kirilowii & - & {$[20]$} \\
\hline & Seeds & T. kirilowii & - & {$[32]$} \\
\hline & Seeds & T. kirilowii & Anti-tumor & {$[38]$} \\
\hline Bryonotic acid (70) & Roots & T. truncata & - & [40] \\
\hline \multicolumn{5}{|l|}{ Flavonoids } \\
\hline Apigenin 7-O- $\beta$-glucopyranoside (71) & Leaves & T. kirilowii & - & {$[41]$} \\
\hline Apigenin 6,8 -di-C- $\beta$-glucopyranoside (72) & Leaves & $\begin{array}{l}\text { T. japonica } \\
\text { T. bracteata } \\
\text { T. cucumerina }\end{array}$ & - & {$[41]$} \\
\hline Luteolin 7-glucoside (73) & Leaves & $\begin{array}{l}\text { T. cucumerina } \\
\text { T. kirilowii }\end{array}$ & - & {$[13]$} \\
\hline Luteolin $3^{\prime}-O-\beta$-D-glucopyranoside (74) & Leaves & $\begin{array}{l}\text { T. japonica } \\
\text { T. bracteata } \\
\text { T. kirilowii }\end{array}$ & - & {$[41]$} \\
\hline Luteolin $4^{\prime}-O-\beta$-D-glucopyranoside (75) & Leaves & $\begin{array}{l}\text { T. japonica } \\
\text { T. kirilowii }\end{array}$ & - & {$[41]$} \\
\hline Kaempferol 3-O- $\beta$-galactopyranoside (76) & Leaves & T. anguina & - & [41] \\
\hline Kaempferol 3-O- $\beta$-sophoroside (77) & Leaves & T. anguina & - & {$[41]$} \\
\hline Quercetin 3-O- $\beta$-rutinoside (78) & Leaves & $\begin{array}{l}\text { T. multilobe } \\
\text { T. rostrata }\end{array}$ & - & {$[41]$} \\
\hline $\begin{array}{l}5,6,6^{\prime} \text {-Trimethoxy- } 3^{\prime}, 4^{\prime} \text {-methylenedioxyisoflavone } 7-O-\beta-\mathrm{D}-\left(2^{\prime \prime} \text { - }\right. \\
O-p \text {-coumaroylglucopyranoside }(79)\end{array}$ & Seeds & T. anguina & - & {$[42]$} \\
\hline \multicolumn{5}{|l|}{ Miscellaneous compounds } \\
\hline Citrulline (80) & Seeds & T. tricuspidata & - & [43] \\
\hline m-Carboxyphenylalanine (81) & Seeds & T. tricuspidata & - & {$[43]$} \\
\hline Punicic acid (82) & Seeds & T. tricuspidata & - & {$[43]$} \\
\hline $\begin{array}{l}(2 R)-(2 \text {-Amino-2-hydroxymethyl-3-[(4-hydroxy-3- } \\
\text { methoxybenzoyl)-O-]-propanoic acid }(\mathbf{8 3})\end{array}$ & Peels & T. kirilowii & - & [44] \\
\hline Methyl 3-(hydroxymethyl)-4-methylbenzoate (84) & Peels & T. kirilowii & - & [44] \\
\hline Vanillc acid (85) & Peels & T. kirilowii & - & [44] \\
\hline Benzyl- $\beta$-D-glucopyranoside (86) & Peels & T. kirilowii & - & [44] \\
\hline$(+)-(7 S, 8 S)$-Guaiacylglycerol-8-O- $\beta$-D-glucopyranoside $(\mathbf{8 7})$ & Peels & T. kirilowii & Anti-inflammation & [44] \\
\hline$\beta$-Carboline (88) & Peels & T. kirilowii & - & {$[44]$} \\
\hline$(3 S)-1,2,3,4$-Tetrahydro- $\beta$-carboline-3-carboxylic acid (89) & Peels & T. kirilowii & Anti-inflammation & [44] \\
\hline Adenosine (90) & Peels & T. kirilowii & - & [44] \\
\hline
\end{tabular}


Table 1 (Continued.)

\begin{tabular}{|c|c|c|c|c|}
\hline Compound & Part of plant & Source & Biological activity & Ref. \\
\hline Guanosine (91) & Peels & T. kirilowii & - & [44] \\
\hline (-)-Loliolide (92) & Peels & T. kirilowii & Anti-inflammation & [44] \\
\hline 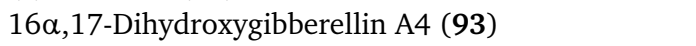 & Peels & T. kirilowii & Anti-inflammation & [44] \\
\hline$(-)-\beta$-Homoarginine anhydride (94) & Roots & T. truncata & Anti-tyrosinase & {$[40]$} \\
\hline 4-Guanidinobutyric acid (95) & Roots & T. truncata & Anti-tyrosinase & [40] \\
\hline 2-Methyl-2-pyridinol (96) & Roots & T. truncata & Anti-tyrosinase & {$[40]$} \\
\hline Nicotinamide (97) & Roots & T. truncata & Anti-tyrosinase & [40] \\
\hline 3-(4-Hydroxyphenyl)propionic acid (98) & Roots & T. truncata & - & {$[40]$} \\
\hline 4-Hydroxybenzoic acid (99) & Roots & T. truncata & Anti-tyrosinase & {$[40]$} \\
\hline 3-Methoxy-4-hydroxybenzoic acid (100) & Roots & T. truncata & $\begin{array}{l}\text { Inhibit ROS } \\
\text { production }\end{array}$ & {$[40]$} \\
\hline Ligballinol (101) & Roots & T. kirilowii & - & {$[21]$} \\
\hline$(10 E, 12 E)-9-O x o-10,12$-octadecadienoic acid (102) & Roots & T. kirilowii & - & {$[21]$} \\
\hline$(9 Z, 11 E)-13-O x o-9,11$-octadecadienoic acid (103) & Roots & T. kirilowii & - & {$[21]$} \\
\hline
\end{tabular}
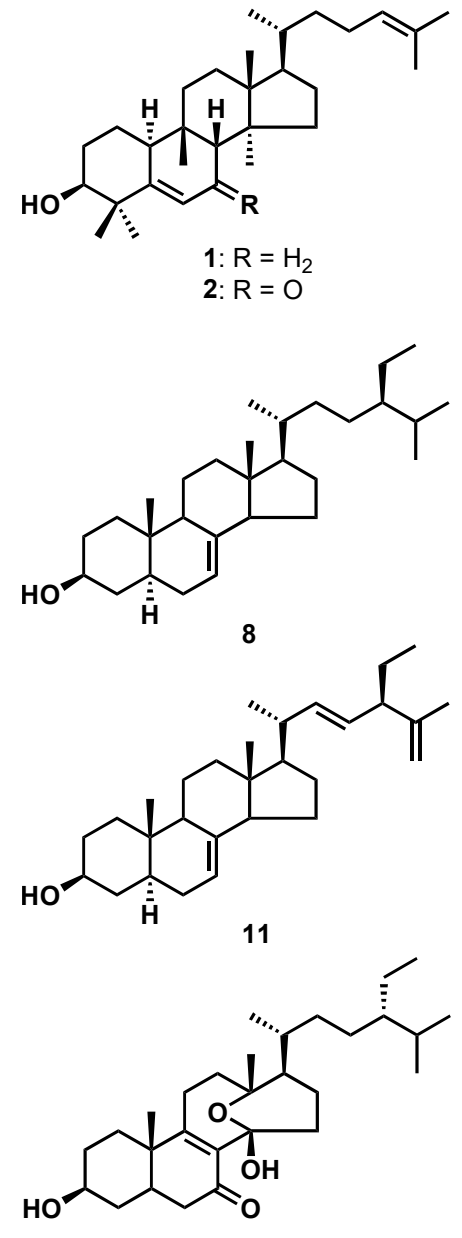

14

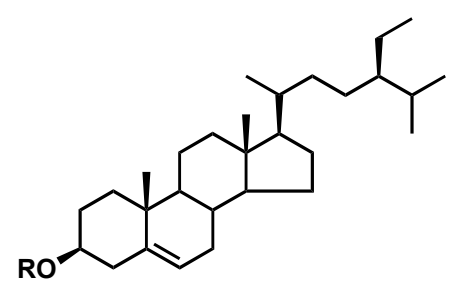

3: $\mathrm{R}=\mathrm{H}$

4: $\mathrm{R}=\mathrm{Glc}$
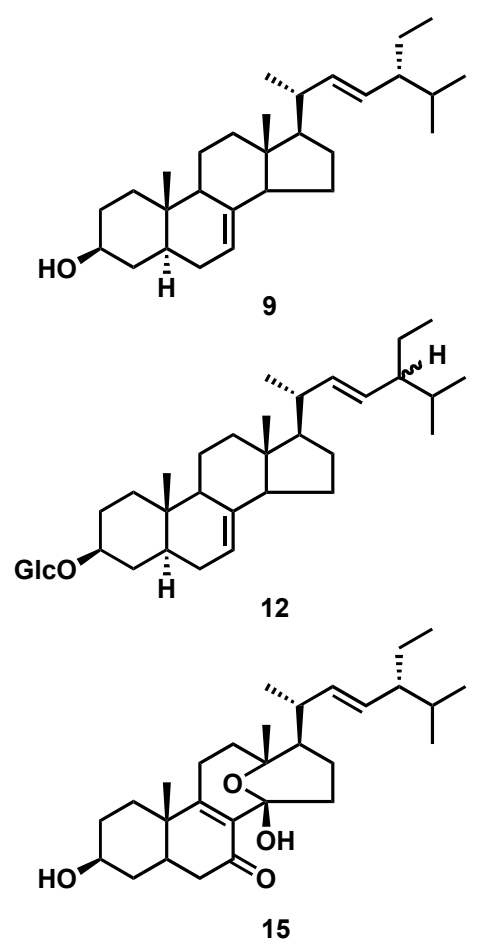<smiles>[R20][C@H]1CC[C@]2(C)[C@@H]3CC[C@@]4(C)[C@@H](CC[C@@H]4[C@H](C)/C=C/[C@H](CC)C(C)C)C3=CC[C@]2(C)C1</smiles>

5: $\mathrm{R}=\mathrm{H}$

6: $R=A c$

7: $\mathrm{R}=\mathrm{Glc}$<smiles>CC[C@H](CC[C@@H](C)[C@H]1CCC2C3=CC[C@H]4C[C@@H](O)CC[C@]4(C)C3CC[C@]21C)C(C)C</smiles><smiles>CC[C@H](CC[C@@H](C)[C@H]1CCC2C3=CC[C@H]4C[C@@H](O)CC[C@]4(C)C3CC[C@@]21C)C(C)C</smiles><smiles>CC1OC(C)C(O)C(O)C(O)C1O</smiles>

Fig. 2 The structures of steroids (1-15) isolated from Trichosanthes species. 

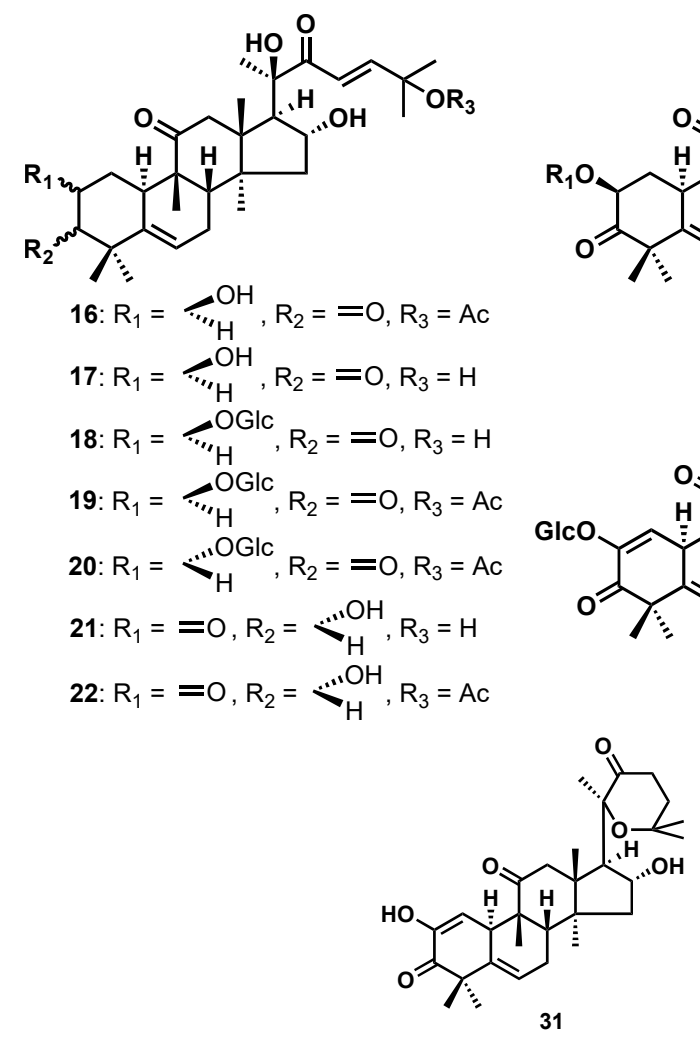
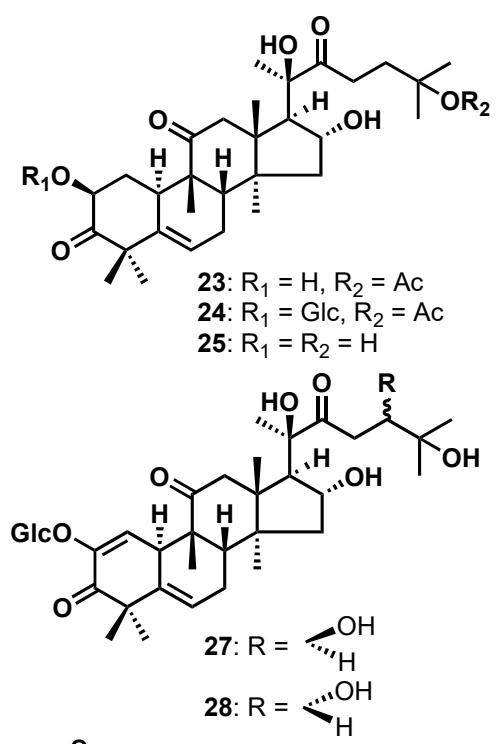
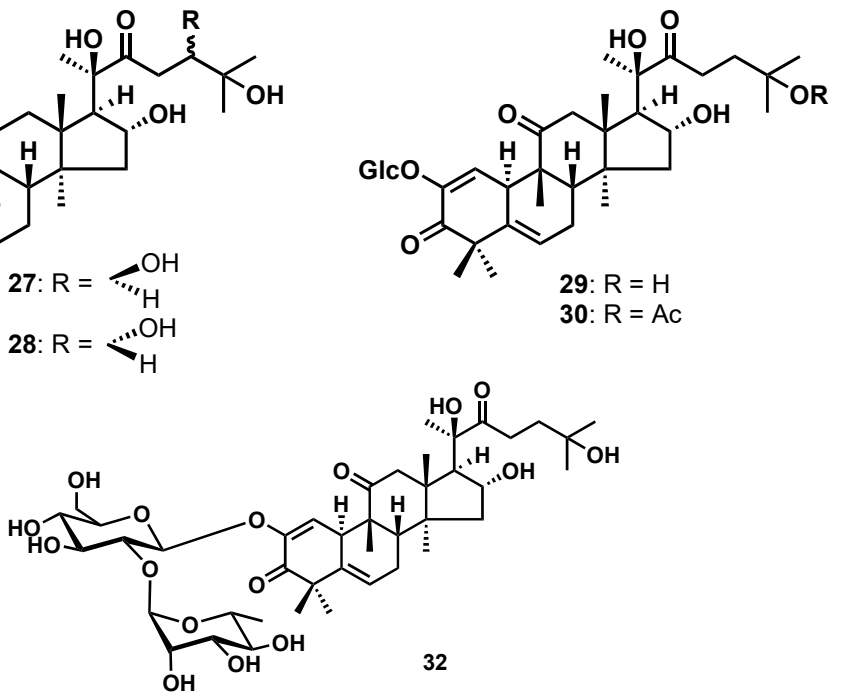

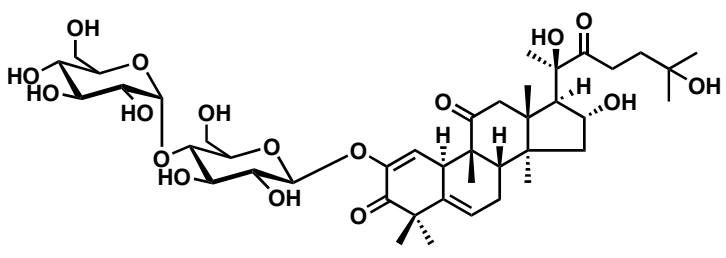

33

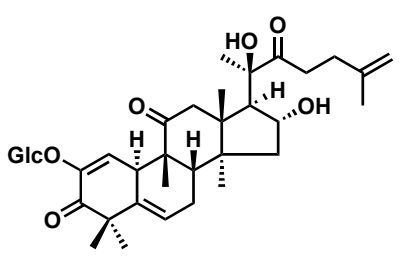

34

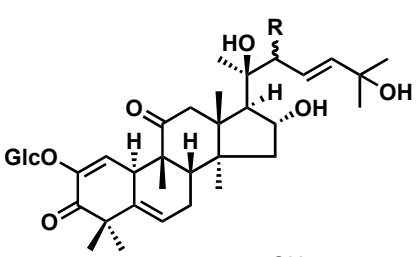

35: $\mathrm{R}={ }_{\cdots, \mathrm{OH}}^{\mathrm{OH}}$ 36: $\mathrm{R}=\stackrel{. \cdots \mathrm{OH}}{\mathrm{H}}$

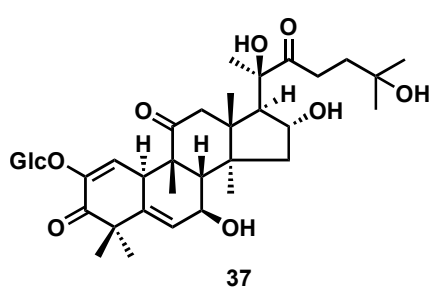
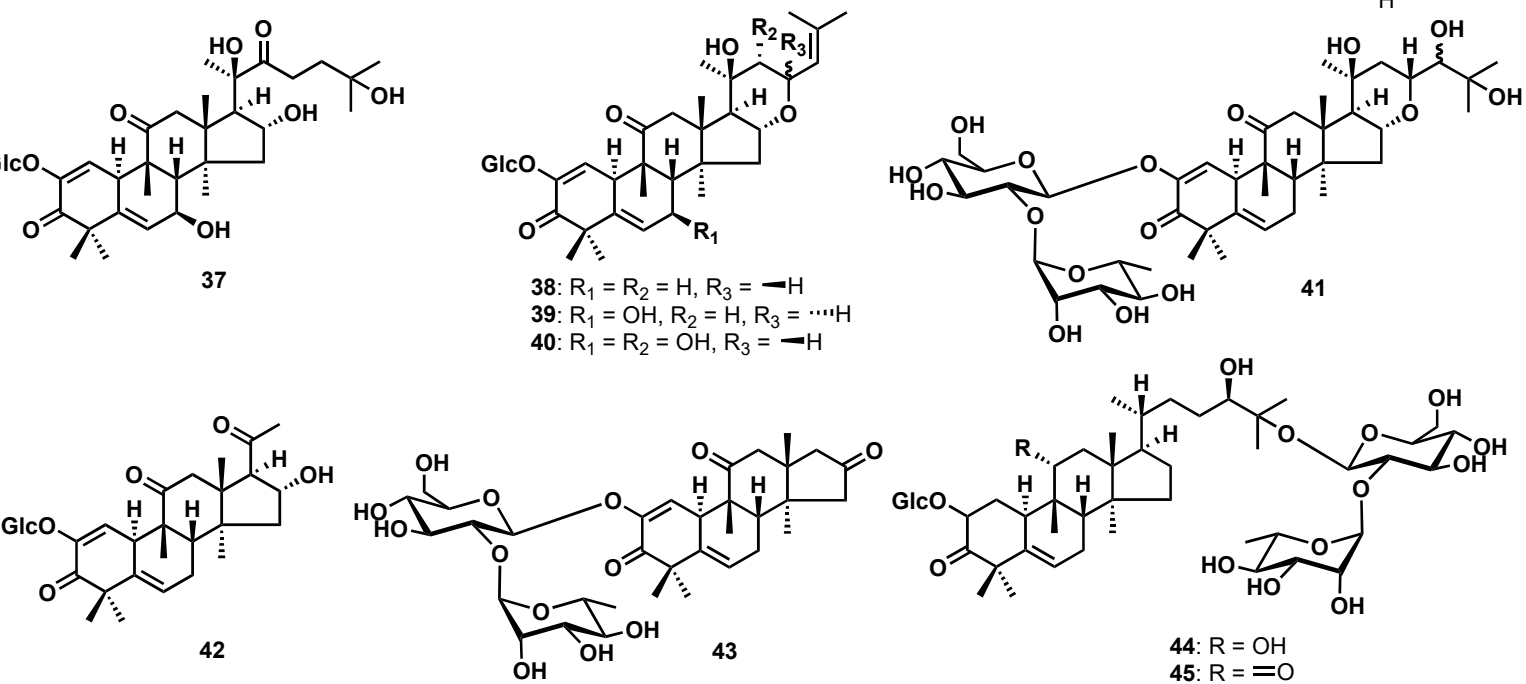

Fig. 3 The structures of triterpenoids (16-70) isolated from Trichosanthes species. 


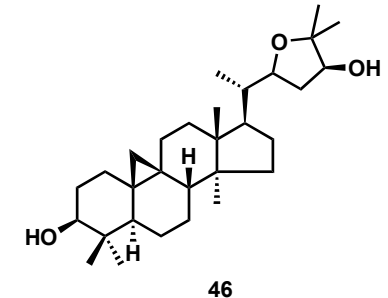

46

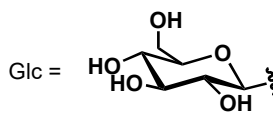

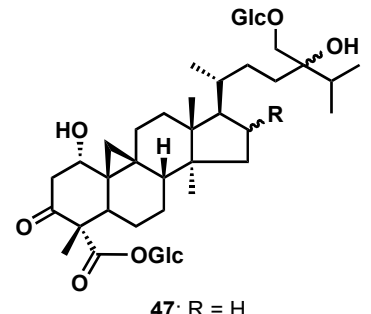

48: $\mathrm{R}=\overbrace{\text { " } \mathrm{OH}}^{\mathrm{OH}}$

49: $\mathrm{R}=\stackrel{.1 \mathrm{OH}}{\mathrm{H}}$

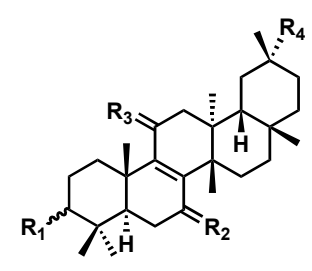

50: $\mathrm{R}_{1}=\mathrm{\prime \prime}_{\mathrm{\prime}}^{\mathrm{OH}}, \mathrm{R}_{2}=\mathrm{R}_{3}=\mathrm{H}_{2}, \mathrm{R}_{4}=\mathrm{CH}_{2} \mathrm{OH}$

51: $\mathrm{R}_{1}=\mathrm{\prime \prime}^{\mathrm{OH}}, \mathrm{R}_{2}=\mathrm{R}_{3}=\mathrm{H}_{2}, \mathrm{R}_{4}=\mathrm{CH}_{2} \mathrm{OAc}$

52: $\mathrm{R}_{1}=\stackrel{\mathrm{OH}}{\mathrm{OH}}, \mathrm{R}_{2}=\mathrm{O}, \mathrm{R}_{3}=\mathrm{H}_{2}, \mathrm{R}_{4}=\mathrm{CH}_{3}$

53: $\mathrm{R}_{1}=\stackrel{\mathrm{OH}}{\mathrm{H}}, \mathrm{R}_{2}=\mathrm{R}_{3}=\mathrm{H}_{2}, \mathrm{R}_{4}=\mathrm{CH}_{2} \mathrm{OH}$

54: $\mathrm{R}_{1}=\stackrel{\because \mathrm{OH}}{\mathrm{H}}, \mathrm{R}_{2}=\mathrm{R}_{3}=\mathrm{H}_{2}, \mathrm{R}_{4}=\mathrm{CH}_{2} \mathrm{OAc}$

55: $\mathrm{R}_{1}=\stackrel{.}{\mathrm{OH}}, \mathrm{R}_{2}=\mathrm{O}, \mathrm{R}_{3}=\mathrm{H}_{2}, \mathrm{R}_{4}=\mathrm{CH}_{2} \mathrm{OH}$

56: $\mathrm{R}_{1}=\stackrel{. \mathrm{OH}}{\mathrm{H}}, \mathrm{R}_{2}=\mathrm{O}, \mathrm{R}_{3}=\stackrel{{ }^{\prime} \mathrm{OH}}{\mathrm{OH}}, \mathrm{R}_{4}=\mathrm{CH}_{2} \mathrm{OH}$

57: $\mathrm{R}_{1}=\stackrel{* \mathrm{OH}}{\mathrm{H}}, \mathrm{R}_{2}=\mathrm{R}_{3}=\mathrm{O}, \mathrm{R}_{4}=\mathrm{CH}_{2} \mathrm{OH}$

58: $\mathrm{R}_{1}=\stackrel{\mathrm{H} H}{\mathrm{O}}, \mathrm{R}_{2}=\stackrel{\mathrm{OH}}{\mathrm{OH}}, \mathrm{R}_{3}=\mathrm{H}_{2}, \mathrm{R}_{4}=\mathrm{CH}_{2} \mathrm{OH}$

70: $\mathrm{R}_{1}=\mathrm{\prime \prime}_{\mathrm{H}}^{\mathrm{OH}}, \mathrm{R}_{2}=\mathrm{R}_{3}=\mathrm{H}_{2}, \mathrm{R}_{4}=\mathrm{COOH}$

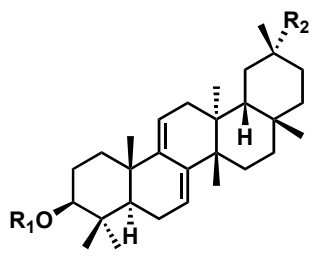

66: $\mathrm{R}_{1}=\mathrm{H}, \mathrm{R}_{2}=\mathrm{CH}_{2} \mathrm{OH}$

67: $\mathrm{R}_{1}=\mathrm{Ac}, \mathrm{R}_{2}=\mathrm{CH}_{2} \mathrm{OAc}$

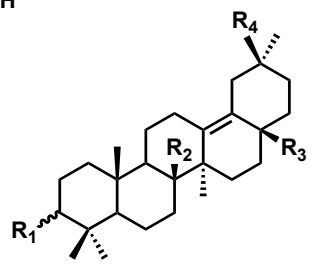

59: $\mathrm{R}_{1}=\mathrm{\prime \prime}^{\mathrm{OH}}, \mathrm{R}_{2}=\mathrm{H}, \mathrm{R}_{3}=\mathrm{COOH}, \mathrm{R}_{4}=\mathrm{CH}_{3}$

60: $\mathrm{R}_{1}=\mathrm{O}, \mathrm{R}_{2}=\mathrm{R}_{3}=\mathrm{CH}_{3}, \mathrm{R}_{4}=\mathrm{COOH}$

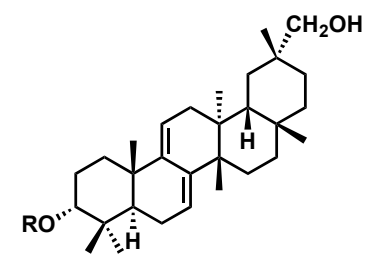

65: $\mathrm{R}=\mathrm{H}=$

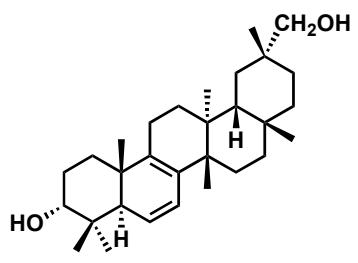

68

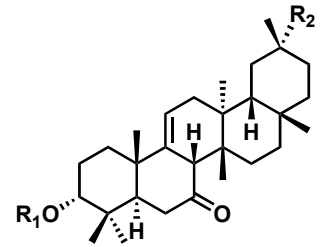

61: $\mathrm{R}_{1}=\mathrm{H}, \mathrm{R}_{2}=\mathrm{CH}_{2} \mathrm{OH}$ 62: $\mathrm{R}_{1}=\mathrm{Ac}, \mathrm{R}_{2}=\mathrm{CH}_{2} \mathrm{OAc}$ 63: $\mathrm{R}_{1}=$

Fig. 3 (Continued.)

against TPA-induced ear inflammation in mice of compounds 51-54 and 61 and 62 with the 50\% inhibitory dose of these triterpenoids in the range of $0.2-0.8 \mathrm{mg} /$ ear has been reported [32]. The biological activities of steroids and triterpenoids are summarized in Table 1.

\section{Flavonoids}

Nine flavonoid glycosides (71-79) were identified from T. anguina, T. bracteata, T. cucumerina, T. japonica, T. kirilowii, T. multilobe and T. rostrata (Fig. 4)
$[13,41,42]$.

\section{Miscellaneous compounds}

Twenty-four other compounds (80-103) have been isolated from T. kirilowii, T. tricuspidata and T. truncata (Fig. 5) [21, 40, 43, 44]. Among them, compounds 87, 89, 92 and 93 showed antiinflammatory activities through inhibition of the activation of NF- $\kappa B$ transcription factors at a concentration of $1 \mu \mathrm{M}$ [44]. Some biological activity of this group of compounds is shown in Table 1. 


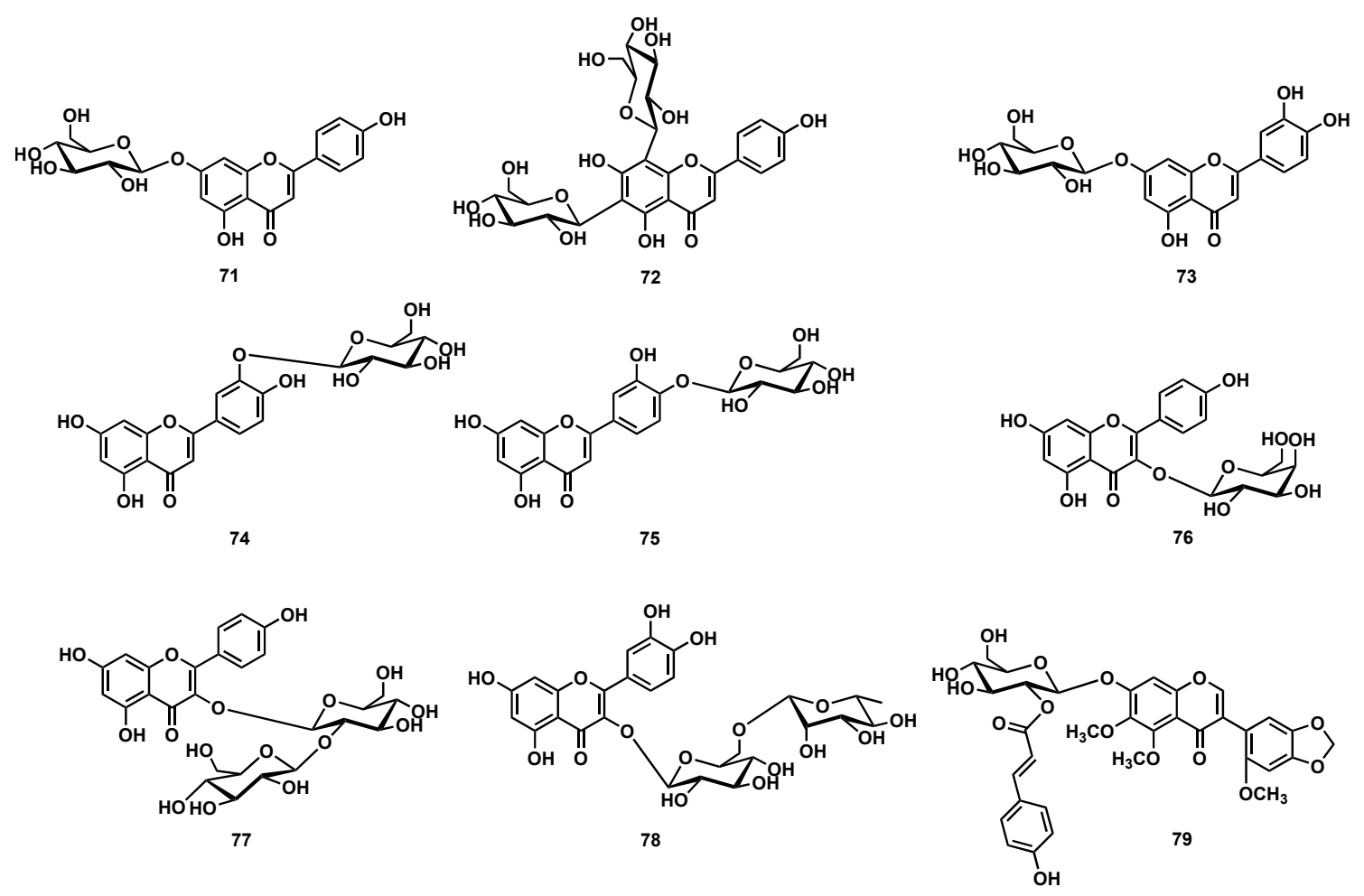

Fig. 4 The structures of flavonoids (71-79) isolated from Trichosanthes species.<smiles>NC(=O)NCCCC(N)C(=O)O</smiles>

80<smiles>COc1cc(C(=O)OCC(N)(C(=O)O)C(C)O)ccc1O</smiles>

83<smiles>c1ccc2c(c1)[nH]c1cnccc12</smiles>

88

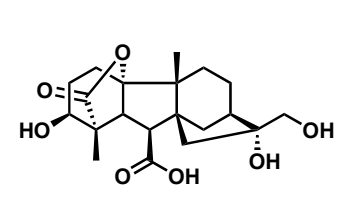

93<smiles>O=C(O)CCCc1cccc(C(=O)O[Na])c1</smiles>

81<smiles>CCCC/C=C\C=C/C=C\CCCCCCCC(=O)O</smiles><smiles>COc1cc(C(O)[C@H](CO)OC2OC(O)C3(O)C(O)C(O)C(O)C(O)C3O2)ccc1O</smiles>

84: $\mathrm{R}_{1}=\mathrm{R}_{3}=\mathrm{CH}_{3}, \mathrm{R}_{2}=\mathrm{CH}_{2} \mathrm{OH}$

85: $R_{1}=H, R_{2}=O^{2} H_{3}, R_{3}=O H$

100: $R_{1}=H, R_{2}=O_{3} H_{3}, R_{3}=O H$

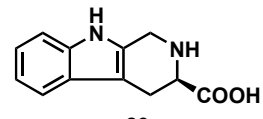

89<smiles>N=C(N)NCCC[C@H](N)CC(=O)OC(=O)C[C@@H](N)CCCNC(=N)N</smiles>

94

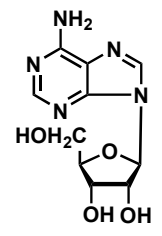

90

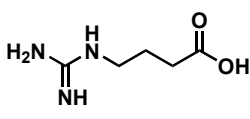

95

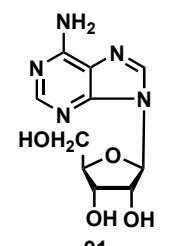<smiles>CC1(C)C[C@H](O)C[C@@H]2OC(=O)C=C21</smiles><smiles>Cc1ncccc1O</smiles>

96<smiles>CC(=O)CCc1ccc(O)cc1</smiles>

97

98<smiles>COC[C@@H]1C(c2ccc(O)cc2)OC[C@H]1c1ccc(O)cc1</smiles>

101

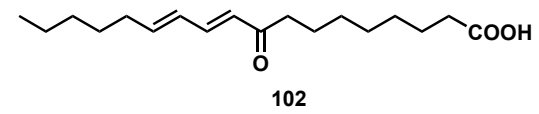

102

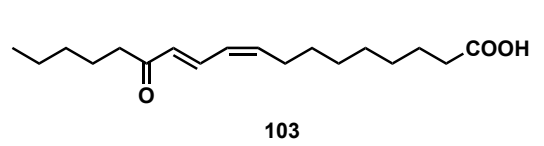

103

Fig. 5 The structures of miscellaneous compounds (80-103) isolated from Trichosanthes species. 


\section{SELECTED BIOLOGICAL ACTIVITIES}

Trichosanthes plants have been used globally as edible and medicinal plants that have shown several pharmacological activities in traditional medicine. Several well-known recent studies have established the therapeutic potential of the plants of this genus as sources of anti-inflammatory, cytotoxic and anticancer, and anti-tyrosinase agents.

\section{Anti-inflammatory activity}

The anti-inflammatory effect of $T$. cucumerina fractions has been reported. Among the tested fractions, methanol and aqueous fractions at a dose of $75 \mathrm{mg} / \mathrm{kg}$ exhibited marked inhibition against carrageenan-induced hind paw edema. The antiinflammatory effect induced by methanol fraction was comparable to that of the reference drug, indomethacin, as well as to the $750 \mathrm{mg} / \mathrm{kg}$ of the fraction at 4 and $5 \mathrm{~h}$ [45]. The anti-inflammatory effect of $50 \%$ ethanol extract of the fruits of T. kirilowii and its effective parts has also been reported. The whole fruit and seed exhibited anti-inflammatory activity against acetic acid-induced vascular permeability in mice, carrageenin-induced edema and cotton pelletinduced granuloma formation in rats, as well as writhing symptoms in mice [46]. In addition, the effects of T. tricuspidata ethanol extract in vitro and in vivo have been studied. The extract attenuated the release of NO and decreased mRNA levels of inducible NO synthase (iNOS), TNF- $\alpha$, and IL- 6 in LPS-induced macrophages and significantly downregulated NF- $\kappa \mathrm{B}$, MAPK, and JAK2 signalling by targeting Syk, Src, and IRAK1 protein kinases. In vivo studies on this extract also produced similar trends in $\mathrm{HCl} / \mathrm{EtOH}$-induced gastritis mouse models by inhibiting proinflammatory cytokines and the inflammatory signalling pathway [47].

\section{Cytotoxic and anti-cancer activities}

One major type of cucurbitacins is a group of natural triterpenoids commonly found in Trichosanthes genus and has long been used in traditional medicine [48-50]. From the recent reports, these triterpenoids have potential and are well-known as new drugs for cancer progression inhibition [51]. Several types of cucurbitacins showed anti-cancer therapeutic properties. For example, cucurbitacin B (16) induces cell cycle in human breast cancer cells, whereas cucurbitacin E (26) inhibits cell proliferation in human prostate cancer cells and causes interruption of the cytoskeleton structure $[52,53]$. In addition, a number of researchers have revealed that cucurbitacin $\mathrm{D}$ (17) induces apoptosis by suppressing the activation of NF- $\mathrm{KB}$ and Stat3 $[54,55]$, induces apoptosis and autophagy in human $\mathrm{T}$ cell leukemia cells [56], and also disturbs viability in MCF7, SKBR3 and MDA-MB 231 breast cancer cells [57]. Furthermore, trichosanthin, a 27$\mathrm{kDa}$ protein isolated from $T$. kirilowii tubers, inhibits breast cancer cell proliferation in both cell lines and nude mice by promotion of apoptosis [58]. In addition, many studies revealed that Trichosanthes plants exhibited anti-tumor activity. For example, it has been reported that the aqueous-alcoholic extract of $T$. dioica root showed anti-tumor and oxidative stress-reducing activity [59]. Moreover, trichosanthin isolated from the root of the same plant has been found to induce apoptosis in tumor cells $[60,61]$. The toxic effects of cucurbitacins prevented the possibility of developing this class of compounds to anti-cancer drugs. However, targeted prodrug design has been proven one of the workable strategies to improve the physicochemical properties of a molecule and overcome unacceptable biopharmaceutical performance. The pharmacological activities of cucurbitacin B (16) have been studied for decades particularly as an anti-tumor activity [62]. Recently, a successful example to convert this highly cytotoxic natural product into potentially useful and relatively less toxic anti-cancer compounds using cellular degradable prodrug design has been reported [63]. Two bioreductive prodrugs, 104 and 105, were synthesized from compound 16 and the study revealed that these prodrugs significantly reduced toxicity against noncancerous cells compared to the parent compound 16 and maintained the original actions against cancer cells. The experiments also confirmed that the prodrugs could efficiently release compound 16 in the reductase-overexpressing MCF7 cells. Among them, the prodrug 104 exhibited significant toxicity reduction in both in vitro and in vivo studies and showed a comparable tumor growth inhibition to that of tamoxifen in the 4T1 xenograft mice experiment (Fig. 6).

\section{Anti-tyrosinase activity}

In the search of new agents from Trichosanthes plants for skin disorders, previous reports revealed that the extracts and their constituents showed anti-tyrosinase activity. For example, compound 100 isolated from T. truncata was shown to dosedependently inhibit ROS production in HaCaT keratinocyte cells without cytotoxicity in the concentration range of $0.2-20 \mu \mathrm{M}$, and compounds 95- 

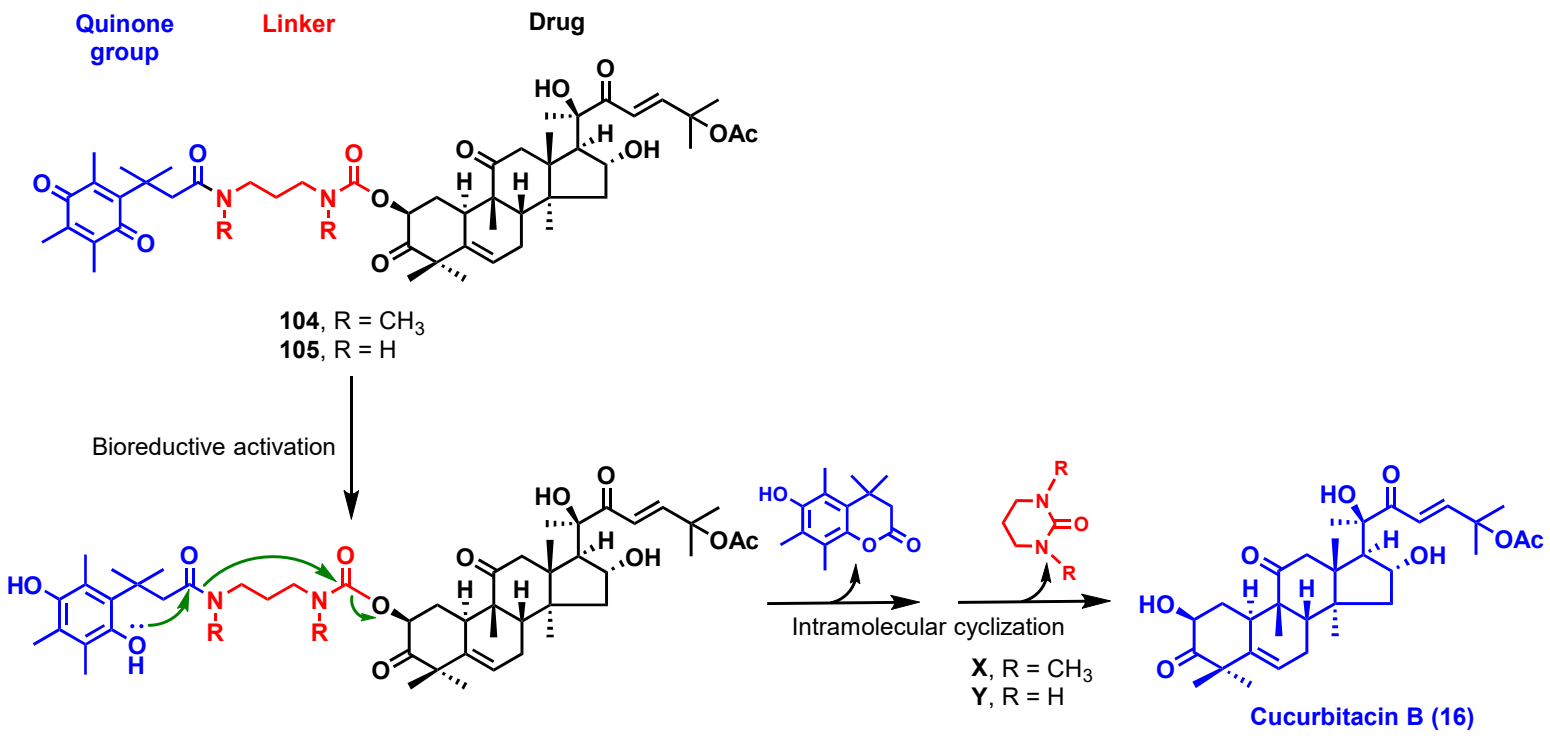

Fig. 6 Rational design of cucurbitacin B-based bioreductive prodrugs [63].

98 and 100 had more potential anti-mushroom tyrosinase activities with $\mathrm{IC}_{50}$ values of 106.9$255.6 \mu \mathrm{M}$ [40]. In addition, the isolated compounds from T. kirilowii pulps have been reported to exhibit tyrosine acidase inhibitory activity [64]. Study on the constituents of the roots of T. kirilowii revealed that cucurbitacin D (2) and 23,24dihydrocucurbitacin $\mathrm{D}$ (25) effectively inhibited the activity of tyrosinase with $\mathrm{IC}_{50}$ of 0.18 and $6.7 \mu \mathrm{M}$, respectively. These compounds also inhibited the synthesis of melanin in B16/F10 melanoma cells, with $\mathrm{IC}_{50}$ of 0.16 and $7.5 \mu \mathrm{M}$, respectively [21].

\section{CONCLUSION}

Trichosanthes is one of the largest genera in the family Cucurbitaceae and plants in this genus are widely used in traditional medicines for treatment of various diseases. The major chemical constituents are steroids, triterpenoids and flavonoids. These compounds exhibited many biological activities and among them are anti-inflammatory, cytotoxic and anti-cancer, and anti-tyrosinase activities. The natural and synthetic, or structurally modified compounds from this plant genus may lead to the discovery of chemical agents with diverse biological activities. Further in-depth pharmacological studies for their potential applications in natural productbased drug discovery are needed.

Acknowledgements: This work was supported by The Thailand Research Fund (grant no. DBG6180030), Ramkhamhaeng University and the Center of Excellence for Innovation in Chemistry (PERCH-CIC), Ministry of Higher Education, Science, Research and Innovation.

\section{REFERENCES}

1. Newman DJ, Cragg GM (2020) Natural products as sources of new drugs over the nearly four decades from 01/1981 to 09/2019. J Nat Prod 83, 770-803.

2. Atanasova AG, Waltenbergerb B, Pferschy-Wenzigc EM, Linder T, Wawrosch C, Uhrin P, Temml V, Wang L, et al (2015) Discovery and resupply of pharmacologically active plant-derived natural products: A review. Biotech Rev 33, 1582-1614.

3. Chomicki G, Schaefer H, Renner SS (2020) Origin and domestication of Cucurbitaceae crops: insights from phylogenies, genomics and archaeology. New Phytologist 226, 1240-1255.

4. Schaefer H, Renner SS (2011) Cucurbitaceae. In: Kubitzki K (ed) The Families and Genera of Vascular Plants, Vol 10, Springer Verlag, Berlin, pp 112-174.

5. Pandey BP (2001) A Textbook of Botany: Angiosperms, S. Chand and Company Ltd., New Delhi.

6. Yang SL, Walters TW (1992) Ethnobotany and the economic role of the Cucurbitaceae of China. Econ Bot 46, 349-367.

7. Dhiman K, Gupta A, Sharma DK, Gill NS, Goyal A (2012) A review on the medicinally important plants of the family Cucurbitaceae. Asian J Clin Nutr 4, $16-26$.

8. Omokhua-Uyi AG, Van Staden J (2020) Phytomedicinal relevance of South African Cucurbitaceae species and their safety assessment: A review. $J$ Ethnopharmacol 259, ID 112967.

9. Rolnik A, Olas B (2020) Vegetables from Cucur- 
bitaceae family and their products; positive effect on human health. Nutrition 2020, ID 110788.

10. Duyfjes BEE, Pruesapan K (2004) The genus Trichosanthes L. (Cucurbitaceae) in Thailand. Thai For Bull (Bot) 32, 76-109.

11. Smitinand T (2014) Thai Plant Names, Rev Edit, Office of the Forest Herbarium, Department of Natural Park, Wildlife and Plant Conservation, Bangkok, pp 564-565.

12. Rekha VPB, Srinivas Gupta BRSS, Anusha P, Venkteswaralu J, Ramesh Babu M, Manideep K (2015) Review on Trichosanthes species: Deserving potential pharmacological properties and boon in medical applications. Am J Ethnomed 2, 303-321.

13. Akihisa $\mathrm{T}$, Yasukawa $\mathrm{K}$, Kimura $\mathrm{Y}$, Takido $\mathrm{M}$, Kokke WCMC, Tamura T (1994) 7-Oxo-10 $\alpha$ cucurbitadienol from the seeds of Trichosanthes kirilowii and its anti-inflammatory effect. Phytochemistry 36, 153-157.

14. Mallavarapu GR, Row LR (1979) Chemical constituents of some Cucurbitaceae plants. Indian $J$ Chem 17B, 417-419.

15. Matsuno T, Nagata S (1971) Sterols from fruits of Trichosanthes cucumeroides and T. japonica. Phytochemistry 10, 1949-1950.

16. Itoh $\mathrm{T}$, Yoshida K, Tamura T, Matsumoto $\mathrm{T}$ (1982) Co-occurrence of C-24 epimeric 24-ethyl- $\Delta^{7}$-sterols in the roots of Trichosanthes japonica. Phytochemistry 21, 727-730.

17. Kanaoka M, Yoshizaki M, Fukino H (1982) Studies on the constituents of Trichosanthes species. I. On the neutral ether extracts of the dried roots of Trichosanthes japonica Regel, Trichosanthes kirilowii Maxim. and Trichosanthes cucumeroides Maxim. Chem Pharm Bull 30, 2570-2574.

18. Jiratchariyakul W, Frahm AW (1989) Isolation and structure elucidation of the substances from Trichosanthes cucumerina L. Mahidol Univ Ann Res 16, 266-267.

19. Sardseangjun C (1993) Chemical composition and pharmacological properties of Trichosanthes cucumerina L. root. Master thesis, Mahidol Univ, Thailand.

20. Akihisa T, Kokke WCMC, Krause JA, Eggleston DS, Katayama S, Kimura Y, Tamura T (1992) 5Dehydrokarounidiol [D:C-friedo-oleana-5,7,9(11)triene-3 $\alpha, 29$-diol], a novel triterpene from Trichosanthes kirilowii Maxim. Chem Pharm Bull 40, 3280-3283.

21. Oh H, Mun YJ, Im SJ, Lee SY, Song HJ, Lee HS, Woo WH (2002) Cucurbitacins from Trichosanthes kirilowii as the inhibitory components on tyrosinase activity and melanin synthesis of B16/F10 melanoma cells. Planta Med 68, 832-833.

22. Silapa-archa W, Picha P, Lurwongrattana O, Kittiwongsunthorn W, Ungsuntnornsarit P (1981) Investigation of the triterpenes of Cucurbitaceae prevalent in Thailand. Mahidol Univ J Pharm Sci 8, 5-8.
23. Ryu SY, Lee SH, Choi SU, Lee CO, No Z, Ahn JW (1994) Antitumor activity of Trichosanthes kirilowii. Arch Pharm Res 17, 348-353.

24. Ha MT, Phan TN, Kim JA, Oh WK, Lee JH, Woo MH, Min BS (2019) Trichosanhemiketal A and B: two 13,14-seco-13,14-epoxyporiferastanes from the root of Trichosanthes kirilowii Maxim. Bioorg Chem 83, 105-110.

25. Minh CV, Nhiem NX, Yen HT, Kiem PV, Tai BH, Anh HLT, Hien TTT, Park SJ, et al (2015) Chemical constituents of Trichosanthes kirilowii and their cytotoxic activities. Arch Pharm Res 38, 1443-1448.

26. Xu Y, Chen G, Lu X, Li ZQ, Su SS, Zhou C, Pei YH (2012) Chemical constituents from Trichosanthes kirilowii Maxim. Biochem Syst Ecol 43, 114-116.

27. Jiratchariyakul W, Frahm AW (1992) Cucurbitacin B and dihydrocucurbitacin B from Trichosanthes cucumerina L. Mahidol Univ J Pharm Sci 19, 5-12.

28. Mai LP, Guénard D, Franck M, Tri MV, Gaspard C, Sévenet T (2002) New cytotoxic cucurbitacins from the pericarps of Trichosanthes tricuspidata fruits. Nat Prod Lett 16, 15-19.

29. Kanchanapoom T, Kasai R, Yamasaki K (2002) Cucurbitane, hexanorcucurbitane and octanorcucurbitane glycosides from fruits of Trichosanthes tricuspidata. Phytochemistry 59, 215-228.

30. Kimura Y, Akihisa T, Yasukawa K, Takase S, Tamura T, Ida Y (1997) Cyclokirilodiol and isocyclokirilodiol: two novel cycloartanes from the seeds of Trichosanthes kirilowii Maxim. Chem Pharm Bull 45, 415-417.

31. Kasai R, Sasaki A, Hashimoto T, Kaneko T, Ohtani K, Yamasaki K (1999) Cycloartane glycosides from Trichosanthes tricuspidata. Phytochemistry 51, 803-808.

32. Akihisa T, Yasukawa K, Kimura Y, Takido M, Kokke WCMC, Tamura T (1994) Five D:C-friedo-oleanane triterpenes from the seeds of Trichosanthes kirilowii Maxim. and their anti-inflammatory effects. Chem Pharm Bull 42, 1101-1105.

33. Akihisa $\mathrm{T}$, Kokke WCMC, Tamura T, Nambara $\mathrm{T}$ (1992) 7-Oxodihydrokarounidiol [7-oxo-D:Cfriedo-olean-8-ene-3 $\alpha, 29$-diol], a novel triterpene from Trichosanthes kirilowii. Chem Pharm Bull 40, 1199-1202.

34. Xu L, Tang CF, Wu C, Ma YC, Chao ZM (2018) A new noroleanane from the seeds of Trichosanthes kirilowii. Nat Prod Commun 13, 813-815.

35. Chao Z, Shibusawa Y, Yanagida A, Shimotakahara S, Shindo H (2005) Two new triterpenes from the seeds of Trichosanthes cucumeroides. Nat Prod Res 19, 211-216.

36. Akihisa T, Kimura Y, Kasahara Y, Kumaki K, Thakur S, Tamura T (1997) 7-Oxodihydrokarounidiol-3benzoate and other triterpenes from the seeds of Cucurbitaceae. Phytochemistry 46, 1261-1266.

37. Akihisa T, Tamura T, Matsumoto T, Eggleston DS, Kokke WCMC, Shimizu N (1988) Karounidiol [D:Cfriedo-oleana-7,9(11)-diene-3 $\alpha, 29$-diol] and its 3- 
$O$-benzoate: novel pentacyclic triterpenes from Trichosanthes kirilowii. X-ray molecular structure of karounidiol diacetate. $J$ Chem Soc Perkin Trans 1 1988, 439-443.

38. Akihisa T, Tokuda H, Ichiishi E, Mukainaka T, Toriumi M, Ukiya M, Yasukawa K, Nishino H (2001) Antitumor promoting effects of multiflorane-type triterpenoids and cytotoxic activity of karounidiol against human cancer cell lines. Cancer Lett 173, 9-14.

39. Akihisa T, Kokke WCMC, Kimura Y, Tamura T (1993) Isokarounidiol [D:C-friedo-oleana-6,8-diene-3 $\alpha, 29$ diol]: the first naturally occurring triterpene with a $\Delta^{6,8}$-conjugated diene system. Iodine-mediated dehydrogenation and isomerization of its diacetate. $J$ Org Chem 58, 1959-1962.

40. Weng I, Lin YA, Chen GY, Chiang HM, Liu YJ, Chen CJ, Lan YH, Lee CL (2018) (-)- $\beta$-Homoarginine anhydride, a new antioxidant and tyrosinase inhibitor, and further active components from Trichosanthes truncata. Nat Prod Res 34, 2262-2268.

41. Yoshizaki M, Fujino H, Masuyama M, Arisawa M, Morita N (1987) A chemotaxonomic study of flavonoids in the leaves of six Trichosanthes species. Phytochemistry 26, 2557-2558.

42. Yadava RN, Syeda Y (1994) Isoflavone glycoside from the seeds of Trichosanthes anguina. Phytochemistry 36, 1519-1521.

43. Dunnill PM, Fowden L (1965) The amino acids of seeds of the Cucurbitaceae. Phytochemistry 4, 933-944.

44. Lei X, Li N, Bai Z, Di J, Zhang H, Dong P, Zhang $P$ (2020) Chemical constituent from the peel of Trichosanthes kirilowii Maxim. and their NF- $\mathrm{BB}$ inhibitory activity. Nat Prod Res, ID 1786825

45. Arawwawala M, Thabrew I, Arambewela L, Handunnetti S (2010) Anti-inflammatory activity of Trichosanthes cucumerina Linn. in rats. $J$ Ethnopharmacol 131, 538-543.

46. Ozaki Y, Xing L, Satake M (1996) Antiinflammatory effect of Trichosanthes kirilowii Maxim. and its effective parts. Biol Pharm Bull 19, 1046-1048.

47. Ahuja A, Jeong D, Kim MY, Cho JY (2019) Trichosanthes tricuspidata Lour. methanol extract exhibits antiinflammatory activity by targeting Syk, Src, and IRAK1 kinase activity. Evid-based Complement Altern Med 2019, ID 6879346.

48. Ríos JL, Escandell JM, Recio MC (2005) New insights into the bioactivity of cucurbitacins. In: Atta-urRahman (ed) Studies in Natural Products Chemistry, Elsevier, Amsterdam, pp 429-469.

49. Kaushik U, Aeri V, Mir SR (2015) Cucurbitacins: an insight into medicinal leads from nature. Pharmacog Rev 9, 12-18.

50. Chen X, Bao J, Guo J, Ding Q, Lu J, Huang M, Wang Y (2012) Biological activities and potential molecular targets of cucurbitacins: a focus on cancer. Anticancer Drugs 23, 777-787.
51. Bartalis J, Halaweish FT (2011) In vitro and QSAR studies of cucurbitacins on HepG2 and HSC-T6 liver cell lines. Bioorg Med Chem 19, 2757-2766.

52. Duangmano S, Sae-lim P, Suksamrarn A, Domann FE, Patmasiriwat P (2012) Cucurbitacin B inhibits human breast cancer cell proliferation through disruption of microtubule polymerization and nucleophosmin/B23 translocation. BMC Complement Altern Med 12, ID 185.

53. Duncan KLK, Duncan MD, Alley MC, Sausville EA (1996) Cucurbitacin E-induced disruption of the actin and vimentin cytoskeleton in prostate carcinoma cells. Biochem Pharmacol 52, 1553-1560.

54. Ku JM, Kim SR, Hong SH, Choi HS, Seo HS, Shin YC, Ko SG (2015) Cucurbitacin D induces cell cycle arrest and apoptosis by inhibiting STAT3 and NF$\kappa \mathrm{B}$ signaling in doxorubicin-resistant human breast carcinoma (MCF7/ADR) cells. Mol Cell Biochem 409, 33-43.

55. Kim SR, Seo HS, Choi HS, Cho SG, Kim YK, Hong EH, Shin YC, Ko SG (2013) Trichosanthes kirilowii ethanol extract and cucurbitacin D inhibit cell growth and induce apoptosis through inhibition of STAT3 activity in breast cancer cells. Evid-based Complement Altern Med 2013, ID 975350.

56. Nakanishi T, Song Y, He C, Wang D, Morita K, Tsukada J, Kanazawa T, Yoshida Y (2016) Autophagy is associated with cucurbitacin D-induced apoptosis in human T cell leukemia cells. Med Oncol 33, ID 30.

57. Ku JM, Hong SH, Kim HI, Lim YS, Lee SJ, Kim M, Seo HS, Shin YC, et al (2018) Cucurbitacin D exhibits its anti-cancer effect in human breast cancer cells by inhibiting Stat3 and Akt signaling. Eur J Inflam 16, 1-9.

58. Fang EF, Zhang CZY, Zhang L, Wong JH, Chan YS, Pan WL, Dan XL, Yin CM, et al (2012). Trichosanthin inhibits breast cancer cell proliferation in both cell lines and nude mice by promotion of apoptosis. PLOS One 7, e41592.

59. Bhattacharya S, Prasanna A, Majumdar P, Kumar RBS, Haldar PK (2011) Antitumor efficacy and amelioration of oxidative stress by Trichosanthes dioica root against Ehrlich ascites carcinoma in mice. Pharmaceut Biol 49, 927-935.

60. Khandaker M, Akter S, Imam MZ (2018) Trichosanthes dioica Roxb.: A vegetable with diverse pharmacological properties. Food Sci Human Wellness 7, 34-48.

61. Sha O, Niu J, Ng TB, Cho EYP, Fu X, Jiang W (2013) Anti-tumor action of trichosanthin, a type 1 ribosome-inactivating protein, employed in traditional Chinese medicine: a mini review. Cancer Chemother Pharmacol 71, 1387-1393.

62. Hunsakunachai N, Nuengchamnong N, Jiratchariyakul W, Kummalue T, Khemawoot $\mathrm{P}$ (2019) Pharmacokinetics of cucurbitacin B from Trichosanthes cucumerina L. in rats. BMC Complement 
Altern Med 19, ID 157.

63. Suebsakwong P, Wang J, Khetkam P, Weerapreeyakul N, Wu J, Du Y, Yao ZJ, Li JX, Suksamrarn A (2019) A bioreductive prodrug of cucurbitacin B significantly inhibits tumor growth in the 4T1 xenograft mice model. ACS Med Chem Lett 10, 1400-1406.

64. Zhang R, Hu X, Zhang B, Wang Z, Hao C, Xin J, Guo Q (2020) Whitening activity of constituents isolated from the Trichosanthes pulp. Evid-based Complement Altern Med 2020, ID 2582579. 